\title{
Multiple hematoma in young patient with sickle cell disease
}

\author{
André Gordinho, Steeve Rosado, Adriana Soares, Maria Antunes \\ Internal Medicine, Hospital Beatriz Ângelo, Loures, Portugal
}

\begin{abstract}
Stroke remains one of the most serious complications of sickle cell disease. Almost $11 \%$ of the patients will have a stroke before the age of 20 . The etiology is hemorrhagic in a small part of the cases, which is associated with a high mortality. We present the case of a 19 year-old patient, with sickle cell disease and multiple associated complications. The patient was admitted with acute chest syndrome and developed a hemorrhagic stroke while hospitalized.
\end{abstract}

Keywords: Sickle cell disease, hemorrhagic stroke, anemia, acute chest syndrome.

Palabras clave: Enfermedad de células falciformes. Ictus hemorrágico. Anemia. Síndrome torácico agudo.

\section{Introduction}

Stroke is one of the most severe complications, and a major cause of sickle cell disease (SCD) morbidity. It affects $3 \%$ to $17 \%$ of all patients with SCD, the prevalence is $11 \%$ in patients under 20 years of age, with a high recurrence rate. Most cases are ischemic, however, hemorrhagic strokes account for $13 \%$ with higher prevalence between 20 and 30 years of age and a substantial higher mortality comparing to ischemic strokes ${ }^{1,2}$. Several risk factors are identified ${ }^{1,3}$, among them: low oxygen blood content, vasculopathy, acute infection, recent acute chest syndrome, classical vascular risk factors (hypertension, diabetes, dyslipidemia), previous stroke or a rapid elevation of hemoglobin.

Although many of the hemorrhagic strokes are subarachnoid hemorrhages after an aneurismal rupture, vasculopathy or even a Moyamoya disease, some cases present with other unexplained etiologies ${ }^{4}$. The standard of care is blood exchange transfusion to achieve hemoglobin $S$ concentration $<30 \%$ and hemoglobin concentration of $10-12 \mathrm{~g} / \mathrm{dl}$, but some studies failed to show benefit in patients with established vasculopathy $y^{4,5}$.

\section{Case Report}

A 19 year-old African man with homozygotic (SS) SCD and multiple associated complications - recurrent priapism, retinopathy, transfusional iron overload, cholecystectomy after lithiasic cholecystitis, multiple vaso-occlusive crisis - was admitted to our emergency department with fever, dyspnea and lower left limb pain. He was compliant with his medication - hydroxyurea $1500 \mathrm{mg}$ per day.

At clinical examination he was febrile (temperature $40.1^{\circ} \mathrm{C}$ ) with increased respiratory effort, tachypnea and low oximetry (89\%) while breathing air. Pulmonary auscultation revealed diffuse diminished respiratory sounds. He was tachycardic with $130 \mathrm{bpm}$ (sinusal rhythm) and his blood pressure was 115/68 mmHg. On his lower left limb, we observed a cicatricial chronic ulcer without signs of ongoing infection.

The first laboratory results revealed a hemoglobin concentration of $8.6 \mathrm{~g} / \mathrm{d}$, normal white blood cell count with neutrophilia (81\%),

\section{Learning points}

- Stroke is one of the major causes of sickle cell morbidity and mortality. - Hemorrhagic strokes are less common (13\% of strokes in SCD patients) but have higher mortality, although risk factors are identified, some cases present with unexplained causes.

- Although some studies failed to show benefit with blood exchange transfusion, it remains the standard of care in stroke prevention.

normal C-reactive-protein $1.3 \mathrm{mg} / \mathrm{dl}$, elevation of hemolytic parameters (total bilirubin $15.18 \mathrm{mg} / \mathrm{dl}$, conjugated bilirubin $2.19 \mathrm{mg} /$ dl, LDH $568 \mathrm{UI} / \mathrm{I}$ ), normal renal function and a blood gas analysis showed severe hypoxemia (p02 $58 \mathrm{mmHg}$, ambient) with hyperlactacidemia (3.7 mmo//). Anteroposterior chest ray revealed a bilateral pulmonary opacity (figure 1 ) and cardiomegaly. A bed-side echocardiography was performed showing normal left and right ventricular function and size, moderate tricuspid regurgitation with a calculated pulmonary artery systolic pressure of $39 \mathrm{mmHg}$.

He was started with intensive IV fluids, analgesic treatment and empiric antibiotics. No anticoagulation nor platelet antiaggregation therapy was given, previously or during his hospitalization.

The patient failed to improve requiring orotracheal intubation and admission to the Intensive Care Unit. He was diagnosed with vaso-occlusive crisis evolving to acute chest syndrome. The repeated laboratory results sustained this clinical course with elevation of inflammatory parameters (leukocytosis $55000 / /, 91 \%$ neutrophilia, c-reactive protein $20 \mathrm{mg} / \mathrm{dl}$ ), lower hemoglobin $6.7 \mathrm{~g} / \mathrm{dll}$ with increased hemolysis (total bilirubin $30.6 \mathrm{mg} / \mathrm{dl}$, conjugated bilirubin $3.1 \mathrm{~g} / \mathrm{dl}$ and LDH 732 U//I) and acute kidney injury (creatinine $2.1 \mathrm{mg} / \mathrm{dl}$, urea $130 \mathrm{mg} / \mathrm{dll}$ ). His hemoglobin S percentage was $81 \%$, a blood exchange transfusion was performed to achieve $\mathrm{HbS}<30 \%$ and hemoglobin of $10 \mathrm{~g} / \mathrm{dl}$.

Figure 1. Chest radiography at admission

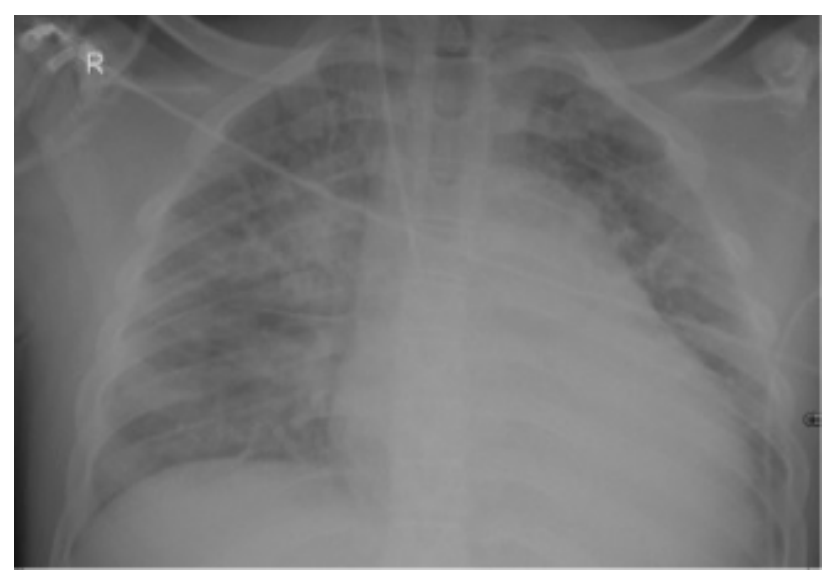




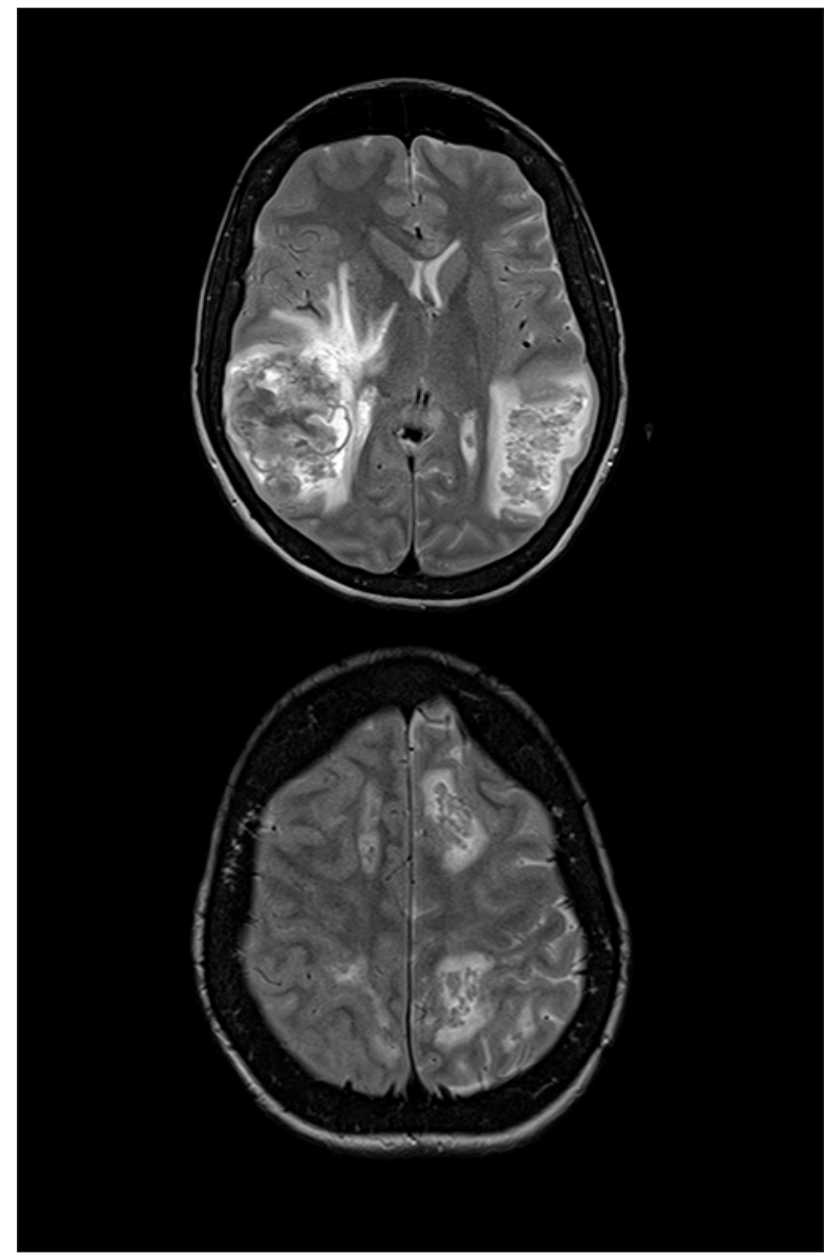

During his hospitalization at the intensive care unit he was mechanically ventilated and given vasopressor agents (norepinephrine) for severe hypotension and septic shock. The patient improved, sedation was stopped daily to assess neurological status. On the 10th day of admission, he was unresponsive and had a hypotonic tetraparesis. His National Institute of Health Stroke score was 33. MRI (figure 2) angiography of the brain showed right $(60 \mathrm{~mm})$ and left $(45 \mathrm{~mm})$ cortico-subcortical parietotemporal hematoma, multiple frontal and occipitotemporal small lesions. The angiography excluded aneurisms, venous sinus thrombosis and Moyamoya disease pattern. He was evaluated by a Neurosurgery team who excluded vasospasm and decided there was no need for surgical intervention and decompression.

The patient recovered slowly, he was extubated and transferred to the Internal Medicine Department 20 days after admission. On the 28th day of hospitalization he was discharged home walking unassisted, he maintained a left hemiparesis grade 4, and modified Rankin scale of 1 (no significant disability despite symptoms, able to carry out all usual duties and activities).

At follow-up 2 months after discharge, he was symptom free. Repeat MRI (figure 3) showed retraction and reduction of the hematoma.

\section{Conclusion}

This case shows one of the most severe complications of sickle cell disease. Hemorrhagic stroke in patients with sickle cell disease has diverse physiopathology. Our patient

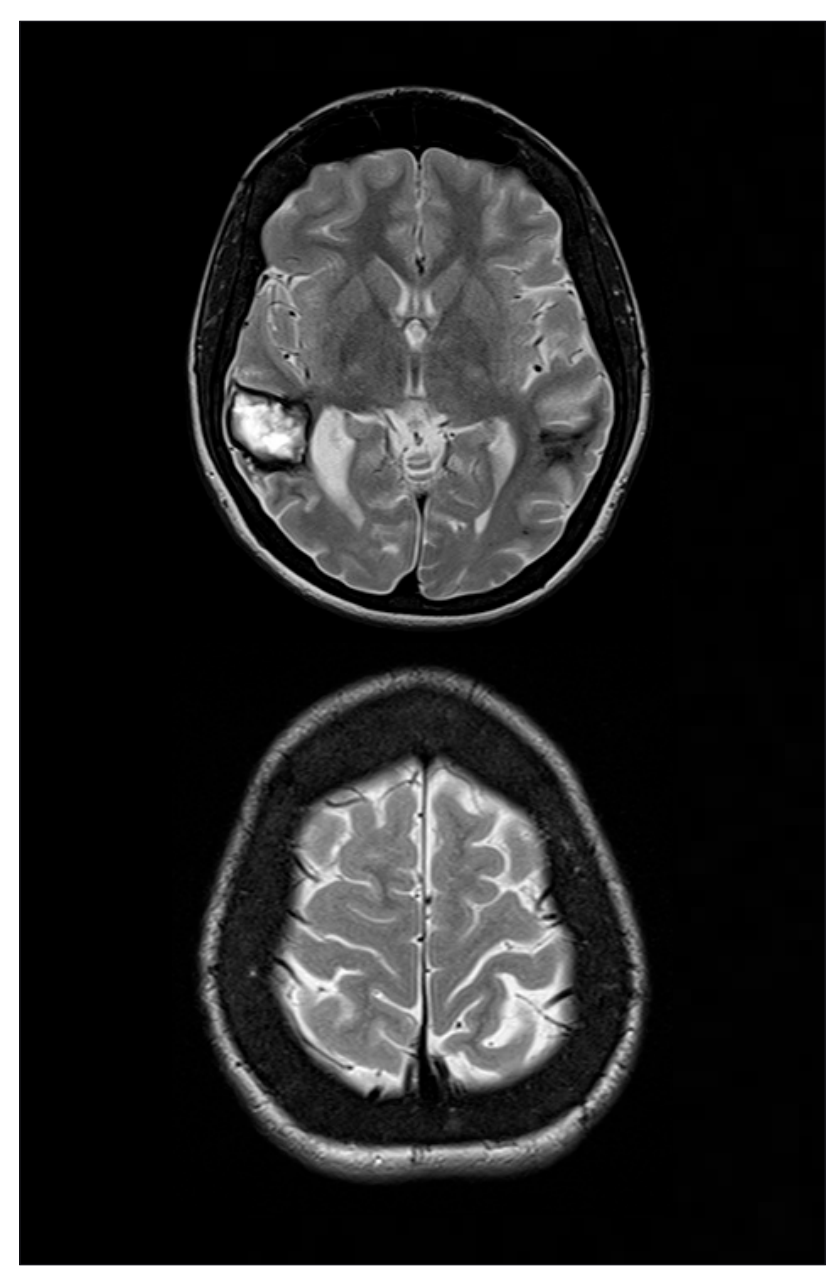

was not hypertensive, he did not have vasospasm, Moyamoya disease or venous sinus thrombosis. In the absence of the most frequent causes, the most likely mechanism is endothelial lesion with wall disruption secondary to vaso-occlusive processes. However, he had some risk factors for hemorrhagic stroke: vasopressor use, recent transfusion and acute chest syndrome ${ }^{3,4}$. He was submitted to blood exchange transfusion according to standard of care which failed to prevent stroke. Despite the severity of the case presented, the patient had a good outcome without any symptoms and was able to carry out all usual activities.

Acknowledgments: Dr. João Espírito Santo, Dr. Francisco Araújo,

Dr. José Lomelino Araújo for their contributions.

\section{References}

1. Menaa F. Stroke in sickle cell anemia patients: A need for multidisciplinary approaches. Atherosclerosis [Internet]. Elsevier Ltd; 2013;229(2):496-503. Available from: http://dx.doi.org/10.1016/i.atherosclerosis.2013.05.006.

2. Webb J, Kwiatkowski JL. Stroke in patients with sickle cell disease. Expert Rev Hematol. 2013;6(3):301-16.

3. Debaun MR, Kirkham FJ. Central nervous system complications and management in sickle cell disease. Blood J. 2016;127(7):829-39.

4. Gueguen A, Mahevas M, Nzouakou R, Hosseini H, Habibi A, Bachir D, et al. Sickle-cell disease stroke throughout life: A retrospective study in an adult referral center. Am J Hematol. 2014;89(3):267-72.

5. Kassim AA, Galadanci NA, Pruthi S, DeBaun MR. How $i$ treat and manage strokes in sickle cell disease. Blood. 2015;125(22):3401-10. 